\title{
A Cryptographic Moving-Knife Cake-Cutting Protocol with High Social Surplus
}

\author{
Yoshifumi Manabe $^{1, a)}$ Risako Otsuka $^{1}$ Tatsuaki Okamoto ${ }^{2, b)}$ \\ Received: July 29, 2014, Accepted: January 7, 2015
}

\begin{abstract}
This paper proposes a cake-cutting protocol using cryptography when the cake is a heterogeneous good that is represented by an interval on a real line. Although the Dubins-Spanier moving-knife protocol with one knife achieves simple fairness and truthfulness, all players must execute the protocol synchronously. Thus, the protocol cannot be executed on asynchronous networks such as the Internet. We show that the moving-knife protocol can be executed approximately but asynchronously by a discrete protocol using a secure auction protocol. The number of cuts is $n-1$ where $n$ is the number of players, which is the minimum. Sgall and Woeginger proposed another asynchronous protocol that satisfies simple fairness, truthfulness, and the minimum number of cuts. These two protocols are compared from the viewpoint of social surplus. The simulation result shows that the cryptographic moving-knife protocol is better than the Sgall-Woeginger protocol.
\end{abstract}

Keywords: game-theory, cake-cutting, moving-knife, secure auction, social surplus

\section{Introduction}

Cake-cutting is an old problem in game theory [2], [16], [18]. It can be employed for such purposes as dividing territory of a conquered island or assigning jobs to members of a group.

This paper discusses achieving a moving-knife protocol using cryptography in cake-cutting when the cake is a heterogeneous good that is represented by an interval, [0,1], on a real line.

The moving-knife protocol is a common technique for achieving fair cake-cutting. The trusted third party (TTP) or one of the players moves a knife on the cake. Every player watches the movement and calls 'stop' when the knife comes to some specific point that is desirable for the player. Cake is cut at the points the calls are made. Many protocols that use one or more knives were shown to achieve some desirable property such as exact division [2].

The simplest moving-knife protocol using one knife was proposed by Dubins and Spanier [6]. The protocol achieves simple fairness and it is truthful.

Moving-knife protocols have several disadvantages. First, all players must watch the knife movement simultaneously, thus moving-knife protocols cannot be executed on networks such as the Internet, in which transmission delays cannot be avoided. In addition, moving knives means cutting the cake at an infinite number of places, thus it is considered to be inefficient.

Many discrete protocols have been proposed that achieve simple fairness [9], [12], [19], [21], [22]. Several different models were proposed that concern the allowed types of primitives. The

Kogakuin University, Shinjuku, Tokyo 163-8677, Japan

NTT Secure Platform Laboratories, Musashino, Tokyo 180-8585, Japan

manabe@cc.kogakuin.ac.jp

okamoto.tatsuaki@lab.ntt.co.jp simplest model is just minimizing the number of cuts. Then, the Robertson-Webb model was proposed [18]. In the model, 'cut' and 'eval' operations are allowed. The complexity of the protocol is given by the total number of these two operations.

However, the cake-cutting problem when applied to the simplest model has not yet been completely solved. Discrete versions of the Dubins-Spanier moving-knife protocol considered in Refs. [8], [21] are not truthful.

Sgall and Woeginger [20] proposed an asynchronous protocol that satisfies simple fairness, truthfulness, and the minimum number of cuts. Its assignment result differs from the one of Dubins-Spanier moving-knife protocol. Sgall-Woeginger protocol is static, that is, every player must decide all of its evaluations in advance. On the other hand, Dubins-Spanier moving-knife protocol is dynamic, that is, every player evaluates the currently remaining piece of cake in each round of the protocol. How the assignment results of these static and dynamic protocols differ is important when these protocols are applied to real world problems.

Cryptography is not commonly used in cake-cutting protocols. A commitment protocol [3] is used in meta-envy-free cakecutting protocols [14] for multiple parties to declare simultaneously their respective private values. Complicated cryptographic protocols have not been used for cake-cutting protocols so far.

\subsection{Our Result}

We show a cryptographic cake-cutting protocol that achieves approximate simple fairness with the minimum number of cuts. We use a secure auction protocol that calculates the maximum bid and the winning player while hiding the bid of each player. The protocol output is approximately the same as that of DubinsSpanier moving-knife protocol. The protocol achieves approxi- 
mate simple fairness and it is truthful. Sgall-Woeginger protocol achieves simple fairness, truthfulness, and the minimum number of cuts. We compare these two protocols in the viewpoint of social surplus. Through a simulation, we show that the social surplus of cryptographic moving-knife protocol is better than that of Sgall-Woeginger protocol. Since the cryptographic movingknife protocol is dynamic, each player can obtain more utility than static Sgall-Woeginger protocol. Thus, the former protocol is superior than the latter one.

\section{Preliminaries}

Throughout the paper, the cake is a heterogeneous good that is represented by interval $[0,1]$ on a real line. Each player $P_{i}$ has a utility function, $\mu_{i}$, that has the following three properties.

(1) For any interval $X \subseteq[0,1]$ whose size is not empty, $\mu_{i}(X)>$ 0.

(2) For any $X_{1}$ and $X_{2}$ such that $X_{1} \cap X_{2}=\emptyset, \mu_{i}\left(X_{1} \cup X_{2}\right)=$ $\mu_{i}\left(X_{1}\right)+\mu_{i}\left(X_{2}\right)$.

(3) $\mu_{i}([0,1])=1$.

The tuple of the utility function of $P_{i}(i=1, \ldots, n)$ is denoted as $\left(\mu_{1}, \ldots, \mu_{n}\right)$. Utility functions might differ among players. No player has knowledge of the utility of the other players.

An $n$-player cake-cutting protocol, $f$, assigns several portions of $[0,1]$ to the players such that every portion of $[0,1]$ is assigned to one player. We denote $f_{i}\left(\mu_{1}, \ldots, \mu_{n}\right)$ as the set of portions assigned to player $P_{i}$ by $f$, when the tuple of the utility function is $\left(\mu_{1}, \ldots, \mu_{n}\right)$.

All players are risk-averse, namely they avoid gambling. They try to maximize the worst case utility they can obtain.

A desirable property for cake-cutting protocols is truthfulness. A protocol is truthful if there is no incentive for any player to lie about his utility function. If a player obtains more utility by declaring a false value, the protocol is not robust. For example, consider the simplest cake-cutting protocol 'divide-and-choose.' In this protocol, Divider first cuts the cake into two pieces $[0, x]$ and $[x, 1]$, such that $\mu([0, x])=\mu([x, 1])=1 / 2$ for Divider. Chooser selects the piece she prefers. Divider obtains the remaining piece. Since the utility function of Divider is unknown to Chooser, Divider can lie about his utility function and cut the cake as $\left[0, x^{\prime}\right]$ and $\left[x^{\prime}, 1\right]$, for any $x^{\prime}(\neq x)$. In this case, Chooser might select the piece such that the utility for Divider is more than half and Divider might obtain less than half. Thus, the risk-averse Divider obeys the rule of the protocol and cuts the cake in half. 'Divide-and-choose' is thus truthful for risk-averse players.

Several desirable properties of cake-cutting protocols have been defined [18]. Simple fairness, which is the most fundamental one, is defined as follows.

For any $i, \mu_{i}\left(f_{i}\left(\mu_{1}, \ldots, \mu_{n}\right)\right) \geq 1 / n$.

This paper discusses simple fair cake-cutting protocols. One of the other types of the desirable property is the social surplus, that is, the total utilities the players obtain. For two protocol $f$ and $f^{\prime}$ which has the same properties (for example, both truthful and simple fair), $f$ is better than $f^{\prime}$ in the sense of social surplus if $\sum_{i=1}^{n} \mu_{i}\left(f_{i}\left(\mu_{1}, \ldots, \mu_{n}\right)\right)>\sum_{i=1}^{n} \mu_{i}\left(f_{i}^{\prime}\left(\mu_{1}, \ldots, \mu_{n}\right)\right)$.

Several kinds of complexity models of discrete cake-cutting problems are defined. The simplest model is that the complexity is the total number of cuts. This model is further divided into two categories.

- Cut-and-calculate model: Any operation that uses the utility function of each player is possible other than cutting.

- Cut-only model: No operation other than cutting is allowed. Thus, the utility of player $P_{i}$ can be known only by $P_{i}$ performing a cut.

Another model called the Robertson-Webb model [18] is introduced. The operations are restricted to the following two types in the model.

- $\operatorname{Cut}_{i}(I, \alpha)$ : Player $P_{i}$ cuts interval $I=\left[x_{1}, x_{2}\right]$ such that $\mu_{i}\left(\left[x_{1}, y\right]\right)=\alpha \mu_{i}(I)$, where $0 \leq \alpha \leq 1$.

- $\operatorname{Eval}_{i}(I)$ : Player $P_{i}$ evaluates interval $I=\left[x_{1}, x_{2}\right]$, which is one of the cuts previously performed using the protocol. $P_{i}$ returns $\mu_{i}(I)$.

The complexity of the Robertson-Webb model is defined as follows.

- Robertson-Webb cut-complexity model: The complexity is measured by the number of cuts. That is, evaluation queries can be issued for free.

- Robertson-Webb cut-and-query-complexity model: The complexity is measured by the total number of cuts and queries.

For the cut-and-calculate model, the minimum number of cuts for simple fair division is $n-1$, where $n$ is the number of players. For the cut-only model, when the number of players is $n=3$, the minimum number of cuts for simple fair division is three [18]. When $n=4$, the minimum number of cuts is four [9]. For a general number of players, the Divide and Conquer protocol [9] achieves $1+n k-2^{k}$ cuts, where $k=\left\lfloor\log _{2} n\right\rfloor[17]$. The lower bound of the cut-only model is $\Omega(n \log n)$ [4].

For the Robertson-Webb cut-and-query-complexity model, the lower bound is $\Omega(n \log n)$ [20]. Edmonds and Pruhs extended the $\Omega(n \log n)$ lower bound to the cases when a player obtains a union of intervals and approximate fairness is achieved [7].

This paper considers the simplest cut-and-calculate model.

\section{Dubins-Spanier Moving-knife Protocol}

This section outlines the Dubins-Spanier moving-knife protocol [6] shown in Fig. 1.

When the number of remaining players is $k$ and the remaining

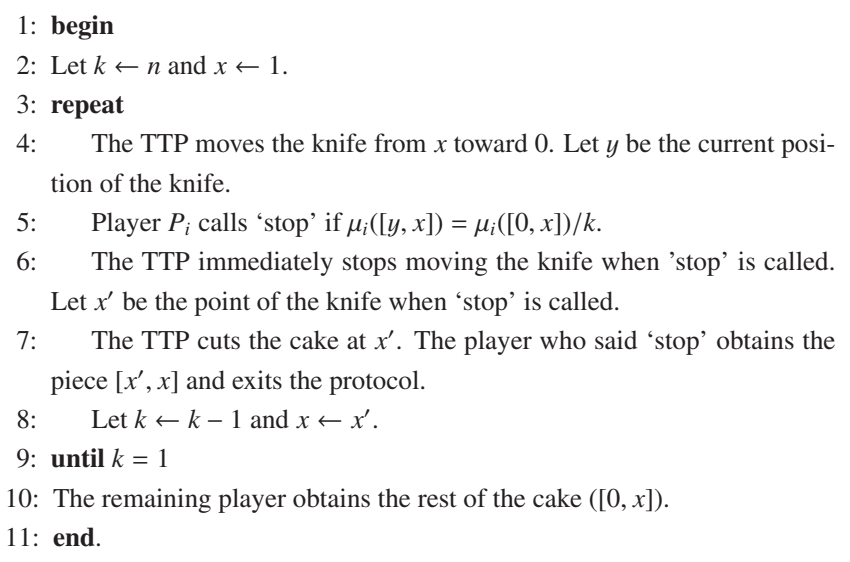

Fig. 1 Dubins-Spanier moving-knife protocol. 


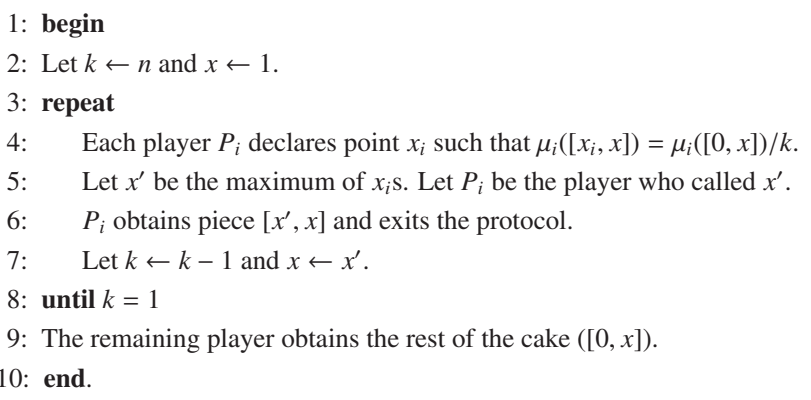

Fig. 2 Endriss protocol.

cake is $[0, x]$, each remaining player $P_{i}$ calls 'stop' if the knife comes to point $y$ which satisfies $\mu_{i}([y, x])=\mu_{i}([0, x]) / k$, that is, the value of piece $[y, x]$ is $1 / k$ of the remaining cake. The first player who calls 'stop' obtains piece $[y, x]$ and exits the protocol. The remaining players continue the same procedure for the remaining cake $[0, y]$.

Each player obtains at least $1 / n$ based on the utility function of the player, thus simple fairness is achieved.

In addition, the protocol is truthful for risk-averse players. Consider the case when player $P_{i}$ tells a lie. Assume that the number of current remaining players is $k$. Let the remaining players be $P_{i}, P_{i+1}, \ldots, P_{i+k-1}$ and the remaining cake be $[0, x]$. The actual place that $P_{i}$ to call 'stop' is $x_{i}$, that is, $\mu_{i}\left(\left[x_{i}, x\right]\right)=\mu_{i}([0, x]) / k$.

If $P_{i}$ calls 'stop' earlier than $x_{i}, P_{i}$ obtains less than $\mu_{i}([0, x]) / k$ and the result is worse than telling the truth.

If $P_{i}$ does not call 'stop' even if the knife comes to $x_{i}$, player $P_{i+1}$ might call 'stop' at $x_{i}-\epsilon$. The remaining piece is $\left[0, x_{i}-\epsilon\right]$ and $\mu_{i}\left(\left[0, x_{i}-\epsilon\right]\right)<(k-1) \mu_{i}([0, x]) / k$. Let $x_{i+1}=x_{i}-\epsilon$. After that, player $P_{j}(j=i+2, i+3, \ldots, i+k-1)$ calls 'stop' at point $x_{j}$ such that $\mu_{i}\left(\left[x_{j}, x_{j-1}\right]\right)=\mu_{i}([0, x]) / k$. If $P_{i}$ calls 'stop' before $x_{j}(j>i+1), P_{i}$ obtains less than $\mu_{i}([0, x]) / k$. If $P_{i}$ does not call 'stop' and obtains the last remaining piece [0, $\left.x_{i+k-1}\right]$, the utility of $P_{i}, \mu_{i}\left(\left[0, x_{i+k-1}\right]\right)$, is less than $\mu_{i}([0, x]) / k$. Therefore, not calling 'stop' at the true point can be worse than telling the truth.

Note that the moving-knife protocol is not a discrete protocol. A protocol is presented by Endriss [8] shown in Fig. 2 that makes the protocol discrete.

It seems that this protocol is the same as the Dubins-Spanier moving-knife protocol, but it is actually not. In this protocol, all players know the cut point of the other players. The cut point information can offer a hint to a player and the player can obtain more utility by behaving dishonestly. Suppose that $n=3$ and the density functions for the utility of the players are as follows.

$$
\begin{aligned}
& u_{1}(z)= \begin{cases}4 / 5 & 0 \leq z \leq 5 / 6 \\
2 & 5 / 6<z \leq 1\end{cases} \\
& u_{2}(z)=1(0 \leq z \leq 1), \\
& u_{3}(z)= \begin{cases}2 & 0 \leq z \leq 1 / 3 \\
1 / 2 & 1 / 3<z \leq 1\end{cases}
\end{aligned}
$$

The utility of $P_{i}$ for $[x, y], \mu_{i}([x, y])$, is calculated by $\int_{x}^{y} u_{i}(z) d z$. Since $\int_{0}^{1} u_{i}(z) d z=1(i=1,2,3)$, these density functions satisfy the conditions of the utility functions.

At the first round, each player declares $c_{1}=5 / 6, c_{2}=2 / 3$,

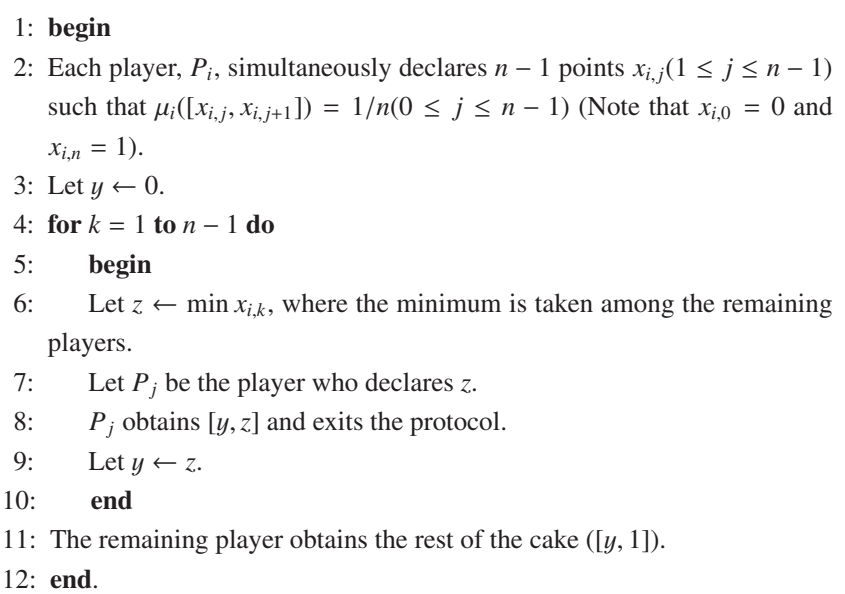

Fig. 3 Sgall-Woeginger protocol.

and $c_{3}=1 / 3$, since $\int_{5 / 6}^{1} u_{1}(z) d z=1 / 3, \int_{2 / 3}^{1} u_{2}(z) d z=1 / 3$, and $\int_{1 / 3}^{1} u_{3}(z) d z=1 / 3$. Since $5 / 6>2 / 3>1 / 3, P_{1}$ obtains $[5 / 6,1]$ and exits the protocol. The next round is performed by $P_{2}$ and $P_{3}$ with the remaining cake $[0,5 / 6]$. The honest declaration, $c_{2}^{\prime}$, at the next round by $P_{2}$ is $5 / 12$, since $\int_{5 / 12}^{5 / 6} u_{2}(z) d z=$ $1 / 2 \int_{0}^{5 / 6} u_{2}(z) d z=5 / 12$. Since $\int_{11 / 48}^{5 / 6} u_{3}(z) d z=1 / 2 \int_{0}^{5 / 6} u_{3}(z) d z$, $P_{3}$ will declare $11 / 48$ as the cut point $c_{3}^{\prime}$, for the next round.

Although $P_{2}$ cannot know $c_{3}^{\prime}$ in advance, it knows that $c_{3}^{\prime}<c_{3}$ is satisfied for any utility function. Thus, $P_{2}$ can declare a false value $1 / 3\left(=c_{3}\right)$, instead of the true value of $5 / 12$ as $c_{2}^{\prime}$, if $P_{2}$ knows that the declared value by $P_{3}$ in previous round is $c_{3}$. When $P_{2}$ declares false value $1 / 3, P_{2}$ wins in this round and obtains $[1 / 3,5 / 6]$. The utility of $P_{2}$ is $1 / 2$, which is larger than utility 5/12 when $P_{2}$ declares the true cut point, 5/12.

Thus knowledge of the declared values of other players destroys the truthful characteristic of the protocol. The trimming protocol [21], which also achieves simple fairness by a discrete protocol, has the same problem about truthfulness, since a player might be able to know all other players' cut points in the previous round.

Sgall and Woeginger [20] showed an asynchronous protocol in which the number of cuts is $n-1$, shown in Fig. 3 .

This protocol achieves simple fairness. When $k=1$, player $P_{i}$ who obtains piece $[0, z]$ satisfies $z=x_{i, 1}$, thus $\mu_{i}\left(\left[0, x_{i, 1}\right]\right)=1 / n$. Next consider the case $k>1$. If player $P_{i}$ obtains $\left[y, x_{i, k}\right]$ in the $k$-th round, $P_{i}$ could not obtain its piece in the previous round. Thus, $y \leq x_{i, k-1}$ is satisfied for for any currently remaining player $P_{i}$ at line 6 and $\mu_{i}\left(\left[y, x_{i, k}\right]\right) \geq \mu_{i}\left(\left[x_{i, k-1}, x_{i, k}\right]\right)=1 / n$.

Since all players declare their cut points simultaneously, no player can know the other players' cut points in advance. Thus, telling a false value such as in the Endriss protocol is not effective in this protocol. Thus the protocol is truthful.

The difference between the Dubins-Spanier moving-knife protocol and the Sgall-Woeginger protocol is the time when the cut points are declared. The latter protocol is static, that is, every player must decide its cut points in advance. The former protocol is dynamic, that is, each player decides his cut point for currently remaining cake in each round. 


\section{Cryptographic Moving-knife Protocol}

The important characteristics of the Dubins-Spanier movingknife protocol are that (1) the declaration is done round by round and (2) when a player $P$ calls 'stop', no player knows the other remaining players' cut points because the knife is moving so that the size of the cutting piece increases.

The simplest solution to keep the protocol truthful and make the protocol discrete would be to have a TTP. In each round, every remaining player privately sends its cut point to the TTP. The TTP decides the largest value and the player who gave the maximum value from the cut point information.

However, it might be difficult to have such a TTP. There might be collusion between a player and the TTP. The TTP might send the player cut point information to the colluding player.

In order to address this problem, we introduce a secure auction protocol. Secure auction protocols have been proposed in cryptography theory [1], [13], [15]. They are outlined as follows.

- Player $P_{i}$ generates its share of public key and secret key, $\left(P K_{i}, S K_{i}\right)$ of a homomorphic encryption scheme.

$P_{i}$ broadcasts $P K_{i}$ and the public encryption key $P K$ is calculated by any player from $\left(P K_{1}, \ldots, P K_{n}\right)$.

$S K_{i}$ is the private key of $P_{i}$ for decryption.

Any player can execute encryption procedure Enc using $P K$. The ciphertext obtained by executing Enc on plaintext $m$ is $\operatorname{Enc}(P K, m)$.

If $P_{1}, \ldots, P_{n}$ jointly execute decryption procedure Dec with their private keys $S K_{1}, \ldots, S K_{n}$, they can decrypt $\operatorname{Enc}(P K, m)$ and obtain $m$. That is, $\operatorname{Dec}(\operatorname{Enc}(P K, m)$, $\left.S K_{1}, \ldots, S K_{n}\right)=m$. Note that the decryption can be performed without revealing the value of $S K_{i}$ to any other players.

For any set of players whose size is less than $n$, they cannot decrypt $\operatorname{Enc}(P K, m)$ by themselves.

- $P_{i}$ encrypts his bid $b_{i}$ using the public key, that is, $P_{i}$ calculates $c_{i}=\operatorname{Enc}\left(P K, b_{i}\right)$.

- $P_{1}, \ldots, P_{n}$ jointly calculates $b_{\max }=\max \left(b_{1}, \ldots, b_{n}\right)$ and player $P_{j}$ who bids $b_{\max }$ from $c_{1}, \ldots, c_{n}$ without directly decrypting $c_{1}, \ldots, c_{n}$ using the homomorphic property.

- During execution of the secure auction protocol, each player gives a zero-knowledge proof [11] that the player acts correctly. The proof can be verified by any other player.

The correctness of the obtained highest bid and the winning player is also given as a zero-knowledge proof. The proof can be verified by any player. That is, no player can deny its bid afterwards.

The details are shown in Refs. [1], [13], [15]. Secure auction protocols use a homomorphic encryption, in which addition of encrypted values can be accomplished without decrypting them. Homomorphic encryption has the following properties.

- There exists polynomial time computable operation $\otimes$ and ${ }^{-1}$ as follows. For any two ciphertext $c_{1}=\operatorname{Enc}\left(P K, m_{1}\right)$ and $c_{2}=\operatorname{Enc}\left(P K, m_{2}\right), c_{1} \otimes c_{2} \in \operatorname{Enc}\left(P K, m_{1}+m_{2}\right)$.

For any ciphertext $c=\operatorname{Enc}(P K, m), c^{-1} \in \operatorname{Enc}(P K,-m)$.

- The encryption is semantically secure, that is, the advantage

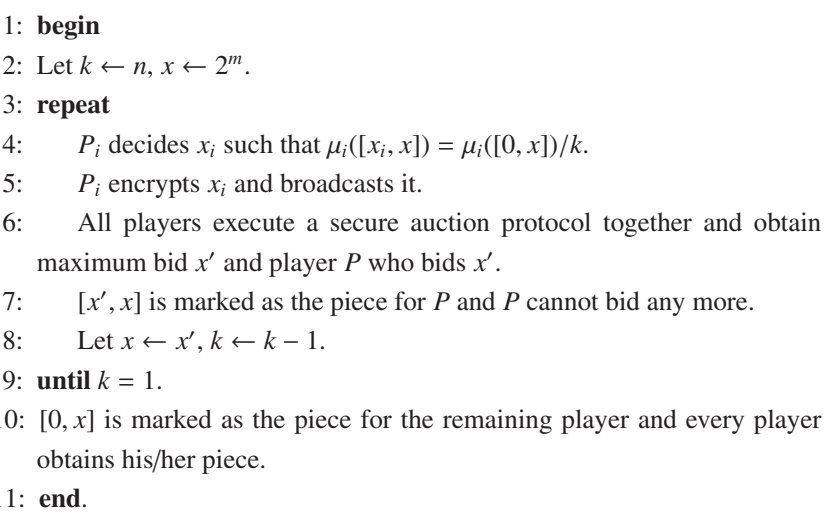

Fig. 4 Cryptographic moving-knife protocol.

of the adversary for the following game is negligible.

The adversary obtains all $P K_{i}$ 's and all $S K_{i}$ 's except for some $S K_{j}$. First, the adversary can repeatedly obtain $\operatorname{Dec}(S K, c)$ for any ciphertext $c$ that it selects. It then outputs two plaintext $m_{0}, m_{1}$. Challenger randomly selects bit $b \leftarrow\{0,1\}$ and $c=\operatorname{Enc}\left(P K, m_{b}\right)$ is given to the adversary.

Then the adversary outputs $b^{\prime}$. It wins if $b=b^{\prime}$

The advantage of the adversary is $\operatorname{Pr}\left[b=b^{\prime}\right]-1 / 2$.

The first property is calculating sum of two ciphertexts without decrypting them. Using the homomorphic characteristics, it is possible to compare multiple bids without decrypting them, that is, they can obtain $C=\operatorname{Enc}\left(P K, \max \left(b_{1}, \ldots, b_{n}\right)\right)$ from $c_{1}, \ldots, c_{n}$. They jointly decrypt $C$ and obtain the maximum bid without knowing each bid. In some secure auction protocol [15], another type of homomorphic encryption scheme is used in which multiplication of two ciphertexts are also possible.

The second property means that no player can obtain information of the plaintext from a given ciphertext if at least one of the secret keys is unknown.

The moving-knife protocol using a secure auction protocol is shown in Fig. 4. In auction protocols, the bids are considered to be an integer. Thus, we convert cake $[0,1]$ to $\left[0,2^{m}\right]$ for some large integer $m$ and each player must bid an integer value for the cutting point. Note that $m$ must be large enough such that for any player $P_{i}$ and any $c \in[0,1], \mu_{i}\left(\left[\left\lfloor c \cdot 2^{m}\right\rfloor / 2^{m}, c\right]\right)$ is negligible, that is, bidding integer values is not a bad approximation.

The protocol is asynchronous, that is, no two events in this protocol need to be executed simultaneously. The number of cuts is $n-1$, which is the minimum.

A difference between the Dubins-Spanier moving-knife protocol and this protocol is that no player exits the protocol during the execution. If a player exits, the set of players who execute the secure auction protocol changes in each round. Changing the set of players requires that the keys be re-generated for the secure auction protocol, thus the protocol would be inefficient. Therefore, the set of players is unchanged in this protocol. However, if a player obtains a piece, the player has no incentive to execute the secure auction protocol honestly any more. Thus, in the proposed protocol, the pieces are actually assigned to the players at the end of the protocol. During the execution of the secure auction protocol, each player presents a proof that the player executes the protocol correctly. If a player misbehaves, it is detected by veri- 


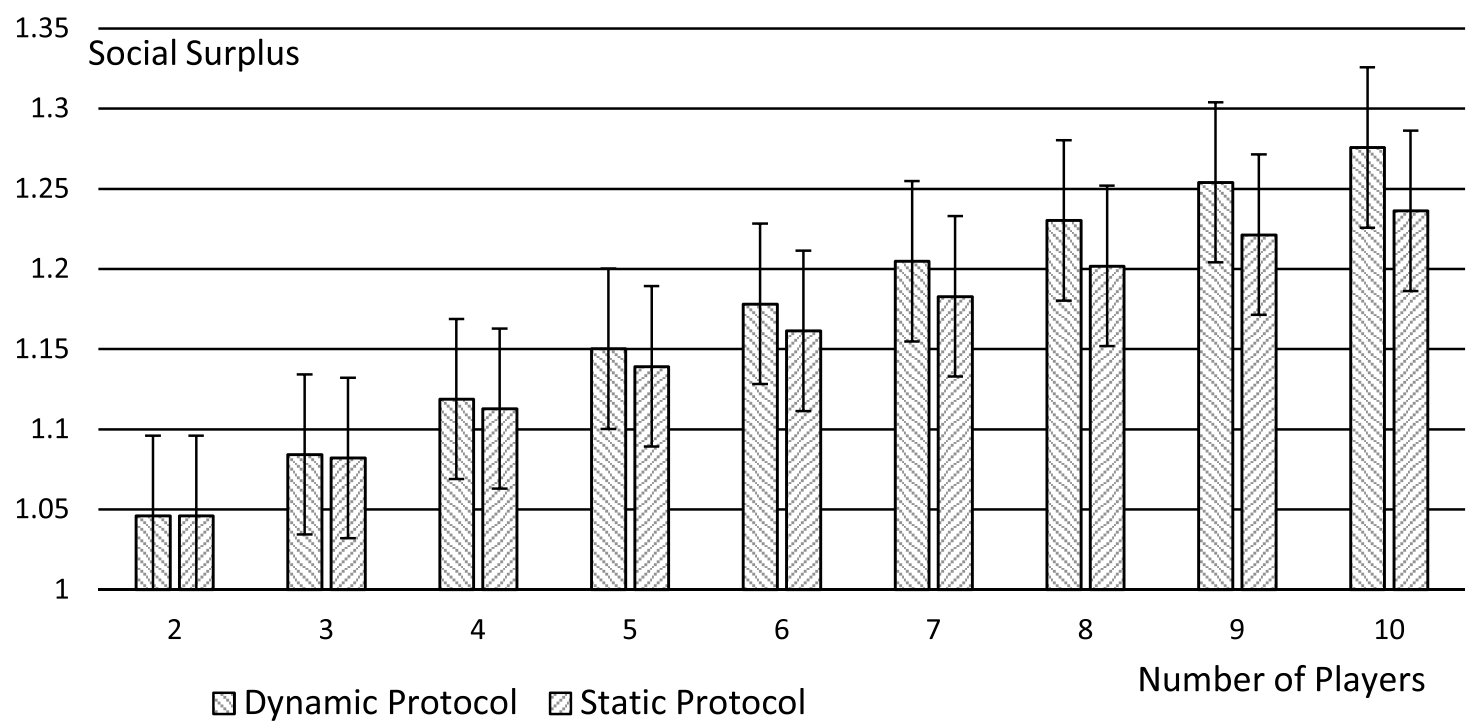

Fig. 5 Social surplus of the two protocols.

fying the proof and the player does not obtain the piece marked for the player. The assignment at the end of the protocol must also be done without TTP. If this protocol is executed just once, there is no way to prevent a player from misbehaving at the assignment without TTP. If this protocol is executed multiple times or some other protocol will be executed among the same players, there is a record of the proof that a player misbehaved in this execution of the protocol, and the player will be rejected from joining another protocol or another execution of this protocol. If a player wants to not be rejected, the player has an incentive to act correctly. A simple way to prove the misbehave is that each player generates a digital signature [10] to the document that records the assignment result given by the protocol. Anyone can check the record by the verification of the digital signatures. If some player misbehaves, then anyone can know the fact by the player's obtained cake and the record with digital signatures.

Simple fairness cannot be achieved by the cryptographic moving-knife protocol because each player bids integer values. We define a cake-cutting protocol $f$ that satisfies approximate simple fairness as follows.

For any $i, \mu_{i}\left(f_{i}\left(\mu_{1}, \ldots, \mu_{n}\right)\right)-1 / n$ is negligible if $\mu_{i}\left(f_{i}\left(\mu_{1}, \ldots, \mu_{n}\right)\right)<1 / n$.

Theorem 1. The protocol in Fig. 4 is truthful for risk-averse players and approximate simple fair. The number of cuts is minimum.

Proof. These properties are achieved because the assignment is an approximation of the one of the Dubins-Spanier moving-knife protocol.

\section{Comparison of Social Surplus}

The difference between Sgall-Woeginger protocol and cryptographic moving-knife protocol is whether the cut point declarations are static or dynamic. The difference affects the assignment results. This section compares these two protocols in the viewpoint of social surplus.

In the cryptographic moving-knife protocol, when $P_{i}$ exits in the first round with obtaining $[x, 1]$, each of the remaining player
$P_{j}$ obtains at least $\mu_{j}([0, x]) /(n-1)$, which is greater than $1 / n$. Since $P_{j}$ did not win in the first round, $\mu_{j}([x, 1])<1 / n$, thus $\mu_{j}([0, x])>(n-1) / n$. Therefore, from the second round, the cake is more than $(n-1) / n$ for the remaining players. The other rounds have the same characteristic. If a player exits with a "small"(in the other players' view) portion of the cake, all of the remaining players obtain more utility.

On the other hand, in the Sgall-Woeginger protocol, when a player exits with a "small" portion of the cake, the extra part of the cake is automatically assigned to the next round's winner. For example, $P_{i}$ wins in the first round and obtains $[0, x]$ and exits, remaining player $P_{j}$ thinks that the remaining cake is $(n-1) / n+\mu_{j}\left(\left[x, x_{j, 1}\right]\right)$, where $\mu_{j}\left(\left[0, x_{j, 1}\right]\right)=1 / n$. In the next round, the player $P_{k}$ wins whose $x_{k, 2}$ is smallest among the remaining players, but the value of the extra part $\mu_{k}\left(\left[x, x_{k, 1}\right]\right)$ might not be large among the remaining players.

In the cryptographic moving-knife protocol, next round call is done for all of the remaining cake, thus the extra part (such as $\left.\left[x, x_{j, 1}\right]\right)$ is also considered by the remaining players. The next round winner is satisfied with a relatively 'small' portion of the cake because of the extra part, thus the next round remaining cake can be larger than in the Sgall-Woeginger protocol. Thus, in the view of the social surplus, the cryptographic moving-knife protocol is more desirable than the Sgall-Woeginger protocol.

We verified the above presumption through a computer simulation. In the simulation, a random utility function is generated for each player as follows. The interval $[0,1]$ is divided into $k$ random intervals $\left[x_{j}, x_{j+1}\right]\left(j=0, \ldots, k, x_{0}=0, x_{k+1}=1\right)$. The utility density function $u_{i}$ for each interval is randomly set between $[0,1] . u_{i}(x)=r_{i}$ for $x_{i} \leq x \leq x_{i+1}$, that is, the utility is uniform for each interval. The utility is normalized such that the total utility $\int_{0}^{1} u_{i}(x) d x=1$. Uniform utility function is commonly used in the discussion of cake-cutting protocols [5], [16] and any random function can be approximated by a set of uniform functions if the intervals are small. In this experiment we set $k=100$ but we obtain similar results for larger and smaller $k(k=10$ and $k=200)$. For the same set of players, static protocol 
(Sgall-Woeginger protocol) and dynamic protocol (cryptographic moving-knife protocol and Endriss protocol with truthful players) are executed. Note that the assignment result of Endriss protocol is just the same as the one of cryptographic moving-knife protocol when every player is truthful.

For each number of players, 3,000 instances are executed. Social surplus is the sum of utilities each player obtained. Figure 5 shows the average of social surplus of 3,000 time experiments when $n$, the number of players, is changed from 2 to 10 . Note that when $n=2$ the assignments are the same. The error bar is standard deviation. According to the t-test there is a significant difference between the mean social surplus by the two protocols for each of $n=3$ to $10(P<0.001)$.

Figure 5 shows the social surplus of cryptographic movingknife protocol is better than that of the Sgall-Woeginger protocol. The difference increases when the number of players increases. The simulation result matches with the above presumption.

\section{Conclusion}

This paper proposed a cryptographic cake-cutting protocol. The protocol is discrete and truthful. It achieves approximate simple fairness with the minimum number of cuts. Its social surplus is better than the Sgall-Woeginger protocol.

Further study will include the use of cryptography in other cake-cutting protocols.

Acknowledgments We thank Dr. Hiro Ito and anonymous referees for their valuable comments. This work was supported by JSPS KAKENHI Grant Number 26330019.

\section{References}

[1] Abe, M. and Suzuki, K.: M+1-st Price Auction Using Homomorphic Encryption, Public Key Cryptography, Naccache, D. and Paillier, P. (Eds.), Lecture Notes in Computer Science, Vol.2274, pp.395-398, Springer Berlin/Heidelberg (online), DOI: 10.1007/3-540-45664-3_8 (2002).

[2] Brams, S. and Taylor, A.: Fair division: From cake-cutting to dispute resolution, Cambridge University Press (1996).

[3] Brassard, G., Chaum, D. and Crépeau, C.: Minimum disclosure proofs of knowledge, J. Comput. Syst. Sci., Vol.37, pp.156-189 (online), DOI: 10.1016/0022-0000(88)90005-0 (1988).

[4] Busch, C., Krishnamoorthy, M.S. and Magdon-Ismail, M.: Hardness Results for Cake Cutting, Bulletin of the EATCS, Vol.86, pp.85-106 (2005).

[5] Chen, Y., Lai, J.K., Parkes, D.C. and Procaccia, A.D.: Truth, justice, and cake cutting, Games and Economic Behavior, Vol.77, No.1, pp.284-297 (2013).

[6] Dubins, L.E. and Spanier, E.H.: How to Cut A Cake Fairly, The American Mathematical Monthly, Vol.68, No.1, pp.1-17 (online), DOI: 10.2307/2311357 (1961).

[7] Edmonds, J. and Pruhs, K.: Cake cutting really is not a piece of cake, Proc. 17th Annual ACM-SIAM Symposium on Discrete Algorithm, SODA '06, pp.271-278, New York, NY, USA, ACM (online), DOI: http://doi.acm.org/10.1145/1109557.1109588 (2006).

[8] Endriss, U.: Cake-Cutting Procedures (2007), available from 〈http:// staff.science.uva.nl//ulle/teaching/comsoc/2007/slides/ comsoc-cakes.pdf $\rangle$.

[9] Even, S. and Paz, A.: A note on cake cutting, Discrete Applied Mathematics, Vol.7, No.3, pp.285-296 (online), DOI: 10.1016/0166218X(84)90005-2 (1984).

[10] Goldreich, O.: Foundations of Cryptography: Volume 1, Basic Tools, Vol.1, Cambridge university press (2001).

[11] Goldreich, O., Micali, S. and Wigderson, A.: Proofs that yield nothing but their validity or all languages in NP have zeroknowledge proof systems, J. ACM, Vol.38, pp.690-728 (online), DOI: http://doi.acm.org/10.1145/116825.116852 (1991).

[12] Kuhn, H.: On Games of Fair Division, Essays in Mathematical Economics in Honor of Oskar Morgenstern, Princeton University Press
(1967).

[13] Kurosawa, K. and Ogata, W.: Bit-Slice Auction Circuit, Proc. 7th European Symposium on Research in Computer Security, ESORICS '02, pp.24-38, London, UK, UK, Springer-Verlag (online), DOI: 10.1007/3-540-45853-0_2 (2002).

[14] Manabe, Y. and Okamoto, T.: Meta-envy-free cake-cutting protocols, Proc. 35th International Conference on Mathematical Foundations of Computer Science, MFCS'10, pp.501-512, Berlin, Heidelberg, Springer-Verlag (online), DOI: 10.1007/978-3-642-15155-2_44 (2010).

[15] Mitsunaga, T., Manabe, Y. and Okamoto, T.: Efficient secure auction protocols based on the Boneh-Goh-Nissim encryption, Proc. 5th International Conference on Advances in Information and Computer Security, IWSEC'10, pp.149-163, Berlin, Heidelberg, Springer-Verlag (online), DOI: 10.1007/978-3-642-16825-3_11 (2010).

[16] Procaccia, A.D.: Cake cutting: Not just child's play, Comm. ACM, Vol.56, No.7, pp.78-87 (2013).

[17] Robertson, J. and Webb, W.: Minimal Number of Cuts for Fair Division, Arts. Comb., Vol.31, pp.191-197 (1991).

[18] Robertson, J. and Webb, W.: Cake-cutting algorithms: Be fair if you can, Ak Peters Series, A.K. Peters (1998).

[19] Saaty, T.: Optimization in integers and related extremal problems, International series in pure and applied mathematics, McGraw-Hill (1970).

[20] Sgall, J. and Woeginger, G.: A Lower Bound for Cake Cutting, Algorithms - ESA 2003, Di Battista, G. and Zwick, U. (Eds.), Lecture Notes in Computer Science, Vol.2832, pp.459-469, Springer Berlin/ Heidelberg (online), DOI: 10.1007/978-3-540-39658-1_42 (2003).

[21] Steinhaus, H.: The Problem of Fair Division, Econometrica, Vol.16, pp.101-104 (1948).

[22] Steinhaus, H.: Mathematical Snapshots, Oxford University Press (1969).

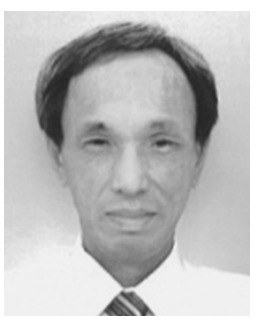

Yoshifumi Manabe was born in 1960. He received his B.E., M.E., and Dr.E. degrees from Osaka University, Osaka, Japan, in 1983, 1985, and 1993, respectively. From 1985 to 2013, he worked for Nippon Telegraph and Telephone Corporation. From 2001 to 2013, he was a guest associate professor of Kyoto University. Since 2013, he has been a professor of Faculty of Informatics, Kogakuin University. His research interests include distributed algorithms, cryptography, game theory, and graph theory. He is a member of IEICE, JSIAM, ACM, and IEEE.

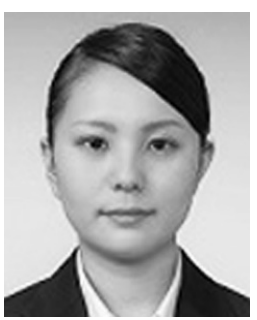

Risako Otsuka received her Bachelor of Informatics from Kogakuin University, Japan, in 2014. Currently, she is a master course student in the graduate school of Systems Design, Kogakuin University. Her research interests include scene metadata generation and usage.

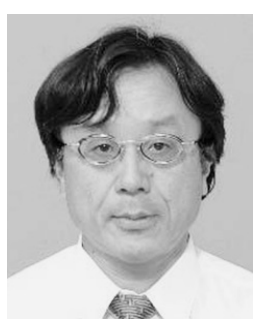

Tatsuaki Okamoto received his B.E., M.E., and Dr.E. degrees from the University of Tokyo, Tokyo, Japan, in 1976, 1978, and 1988, respectively. He is a Fellow of NTT Secure Platform Laboratories. $\mathrm{He}$ is presently engaged in research on cryptography and information security. He is a guest professor of Kyoto

University. 